\title{
Development of a dispersion interferometer for magnetic confinement plasma and its application to atmospheric pressure plasmas
}

\author{
T. Akiyama ${ }^{1, a}$, R. Yasuhara ${ }^{a}$, K. Kawahata ${ }^{a}$, K. Nakayama $^{b}$, S. Okajima ${ }^{\text {, }}$, \\ K. Urabe ${ }^{c, d}, K$. Terashima ${ }^{c}$, and N. Shirai ${ }^{\mathrm{e}}$ \\ ${ }^{a}$ National Institute for Fusion Science, 322-6 Oroshi-cho, Toki, Japan, 509-5292 \\ ${ }^{b}$ Chubu University, 1200 Matsumoto-cho, Kasugai, Japan, 487-8501 \\ ${ }^{c}$ The University of Tokyo, 5-1-5 Kashiwanoha, Kashiwa, Japan, 277-8561 \\ ${ }^{d}$ Japan Society for the Promotion of Science, 5-3-1, Koji-machi, Chiyoda, Japan, 102-0083 \\ ${ }^{e}$ Tokyo Metropolitan University, 1-1 Minami-ohsawa, Hachioji, Japan, 192-0397 \\ E-mail: takiyama@nifs.ac.jp
}

\begin{abstract}
A $\mathrm{CO}_{2}$ laser dispersion interferometer (DI) has been developed for both magnetically fusion plasmas and atmospheric pressure plasmas. The DI measures the phase shift caused by dispersion in a medium. Therefore, it is insensitive to mechanical vibrations and changes in the neutral gas density, which degrade the resolution of the plasma measurement. We installed the DI on the Large Helical Device (LHD) and demonstrated a high density resolution of $2 \times 10^{17} \mathrm{~m}^{-3}$ without a vibration-free bench. This shows good agreement with results of another interferometer. The DI system is applied to the electron density measurement of the atmospheric pressure plasmas, which is smaller than $1 \mathrm{~mm}$. The significant suppression of the phase shift caused by the neutral gas is proven. The achieved density resolution was $1.5 \times 10^{19} \mathrm{~m}^{-3}$ with a response time of $100 \mu \mathrm{s}$.
\end{abstract}

First EPs Conference on Plasma Diagnostics - $1^{\text {st }}$ ECPD

14-17 April 2015

Villa Mondragone, Frascati (Rome) Italy

\footnotetext{
${ }^{1}$ Speaker
} 
1. Introduction

An interferometer is a conventional tool for measuring the electron density of a plasma. It measures the phase difference between a probe and reference lights. The phase difference depends on not only the electron density, but also the change in the optical path length due to mechanical vibrations, and the changes in the neutral gas density. Since the phase shifts caused by the three terms cannot be distinguished in the conventional interferometer, the latter two terms lead to measurement errors.

Use of a vibration-free bench is preferable to reduce the vibrations. A two-color interferometer is the typical method to separate the phase shift due to the electron density from the other phase shifts. It consists of two interferometers with different wavelengths. While the phase shift due to the plasma is proportional to the wavelength, the other phase shifts are inversely proportional. From the different dependences on the wavelength, we can evaluate the electron density by solving simultaneous equations. Although the technique is widely used, the separation is not always perfect. In addition, the optical system becomes somewhat complicated.

\section{2. dDispersion interferometer}

A "dispersion interferometer" (DI) measures the phase difference, which originates from dispersion in a medium, between the fundamental and the second harmonic light. Since the dependence of the refractive index of neutral gas on the wavelength is quite small, the phase shift caused by the neutral gas can be reduced significantly with the DI. The mechanical vibrations change only the path length, and do not change the refractivity. Hence the DI cannot measure the vibration. In this way, the DI is not affected by the vibrations and the neutral gas density, and can selectively measure the dispersion component.

At first, the dispersion interferometer was used for transmission tests of semiconductors and for contouring the surface [1]. Since a plasma has dispersion, the phase shift can be measured with the DI. The DI was implemented to measure the electron density of a plasma of laser spark [2]. At present, some fusion plasma devices are equipped with the dispersion interferometers [3-5].

The optical setup of the DI is as follows. A laser light is injected in a nonlinear crystal and the second harmonic component is generated. The probe beam is a mixture of the fundamental and the second harmonic components, whose beam axes are almost the same. After passing through a plasma, the other nonlinear crystal generates the second harmonic component again. Following a filter to eliminate the fundamental component, the interference signal between two second harmonic components is detected.

The interference signal of the original DI [3] is almost the same as that of a homodyne interferometer $(\mathrm{I}=A+B \cos \theta)$. Hence, the DI must measure the DC 
component $A$ and the $\mathrm{AC}$ component $B$ of the interference signal to evaluate the phase shift. Although the DC and AC components are usually measured without a plasma, they vary during a discharge. This is because they are determined by the detected intensities, and the intensities change during discharges by the beam deviation caused by the electron density gradient, oscillation instabilities of a laser, and electrical noises. The variations of $A$ and $B$ lead to measurement errors. Hence, we introduced a phase modulation technique and a new phase detection method using amplitude of modulation components [6]. Compared to the other dispersion interferometers, this method is free from variations of detective intensities in principle.

\section{Optical setup of dispersion interferometer}

As a laser source, we use a continuous-wave $\mathrm{CO}_{2}$ laser with a wavelength and a power of $10.6 \mu \mathrm{m}$ and $8 \mathrm{~W}$, respectively. $\mathrm{AgGaSe}_{2}$ with a length of $15 \mathrm{~mm}$ is used as the nonlinear crystal. The second harmonic beam with a power of $50 \mu \mathrm{W}$ is obtained from injection of the $8 \mathrm{~W}$ laser. After passing through a plasma, a photoelastic modulator gives a phase modulation with a frequency of $50 \mathrm{kHz}$ to the second harmonic component only. Since the transmissivity of a saphirre at $10.6 \mu \mathrm{m}$ is almost zero, it is used to eliminate the fundamental component. The modulated interference signal of two second harmonic components is detected with a Peltier cooling detector. The electron density can be evaluated from the ratio of modulation amplitudes [6]. The amplitudes of the modulation components are obtained with lock-in amplitude.

\section{Measurement results}

\subsection{Fusion plasma}

We installed the $\mathrm{CO}_{2}$ laser DI [7] on

the Large Helical Device (LHD) at National Institute for Fusion Science. LHD is a superconductor machine with a device diameter of $13.5 \mathrm{~m}$. The achieved density resolution, which is determined by the zero point variations, is $2 \times 10^{17} \mathrm{~m}^{-}$ ${ }^{3}$ for several-seconds measurement and $5 \times 10^{17} \mathrm{~m}^{-3}$ for $30 \mathrm{~min}$ without a vibration isolation system. Since the typical electron density is $10^{19}-10^{20} \mathrm{~m}^{-3}$, the high density resolution is obtained without any vibration isolation bench for the optical components. This result demonstrates that the DI is insensitive to

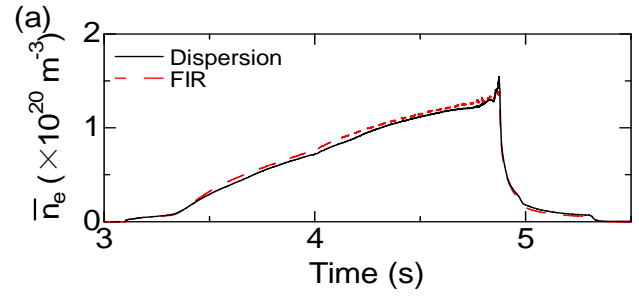

(b)

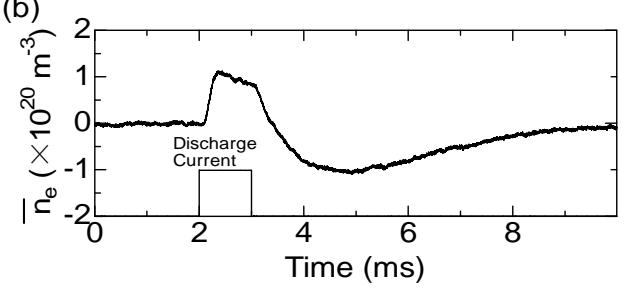

Fig. 1: Measurement results of (a) LHD ( $\mathrm{L}=3.28 \mathrm{~m})$ and (b) atmospheric pressure $(\mathrm{L}=500 \mu \mathrm{m})$ plasmas. the vibrations. Figure 1 (a) shows an example of measurement results of an LHD 
plasma. The measurement agrees with the density measured with the existing farinfrared (FIR) laser interferometer.

\subsection{Atmospheric pressure plasma}

A source of an atmospheric pressure plasma was brought to the LHD hall and the optical setup was modified to measure atmospheric pressure plasmas [8]. A plasma diameter is about $0.5-1 \mathrm{~mm}$ and the working gas is He. It is a pulse operation with a frequency of $100 \mathrm{~Hz}$. A measurement result of an atmospheric pressure plasma with the same system as Fig. 1 (a) is shown in Fig. 1 (b). The temporal resolution was found to be $100 \mu$ s from the response of the plasma start-up. Generally speaking, the phase shift due to changes in the neutral gas density is much larger than that due to plasma in the case of the conventional interferometer. Hence, temporal behaviors of the electron density are masked. The phase shift due to changes in the neutral gas density can be rather suppressed with the DI and the electron density can be evaluated to be $1 \times 10^{20} \mathrm{~m}^{-3}$ from rapid changes in the phase at the beginning and the end of the discharge. Rapid changes at the beginning and end of the discharges are almost the same, thus we assume the electron density is almost constant during the discharge. The gradual changes in the phase difference reflect the changes in the neutral gas density. This is caused by the small dispersion component of the neutral gas. Just after the plasma production, the neutral gas density began to decrease due to expansion of the gas and this continued until even after the plasma termination. After a period of time, the density gradually returned to the initial state. The achieved density resolution is $1.5 \times 10^{19} \mathrm{~m}^{-3}$ for 4096 data average.

\section{Conclusions}

We have developed the $\mathrm{CO}_{2}$ laser dispersion interferometer which uses the ratio of modulation amplitude to measure the electron density of a plasma. On fusion devices, a good density resolution of $2 \times 10^{17} \mathrm{~m}^{-3}$ is achieved without a vibration isolation system. We have applied the dispersion interferometer to atmospheric pressure plasmas. It is demonstrated that the dispersion interferometer can remove the phase shift due to changes in the neutral gas density, which reduces the signal to noise ratio of a conventional interferometer for the atmospheric pressure plasmas.

\section{References}

[1] F. A. Hopf et. al., Opt. Lett. 5, 386 (1980).

[2] Kh. P. Alum et. al., Sov. Tech. Phys. Lett. 7, 581 (1981).

[3] V. P. Drachev et. al., Rev. Sci. Instrum. 64, 1010 (1993). 
Dispersion interferometer for fusion an atmospheric pressure plasmas

[4] H. Dreier et. al., Rev. Sci. Instrum. 82, 063509 (2011).

[5] F. Brandi et. al., Rev. Sci. Instrum. 80, 113501 (2009).

[6] T. Akiyama et. al., Plasma and Fusion Research 5, S1041 (2010).

[7] T. Akiyama et. al., Rev. Sci. Instrum. 85, 11D301 (2014).

[8] K. Urabe et. al., J. Phys. D: Appl. Phys. 47, 262001 (2014). 